\title{
Intraocular Tuberculosis: A Challenging Case Mimicking Wet Age-Related Macular Degeneration
}

\author{
Emanuela Interlandi ${ }^{a}$ Francesco Pellegrini $^{b}$ Carlos Pavesio $^{c}$ \\ Marco De Luca ${ }^{a}$ Rocco De Marco a ${ }^{a}$ Alessandro Papayannis ${ }^{d}$ \\ Erika Mandaràe Alessandra Cuna ${ }^{e}$ Daniele Cirone ${ }^{f}$ Cristina Ciabattoni ${ }^{9}$ \\ Tatiana Liberalib $^{b}$ Antonio Zappacostab ${ }^{b}$ Loredana Latanza $^{\text {h }}$ \\ aDepartment of Ophthalmology, "Ospedale del Mare" Hospital, Naples, Italy; ${ }^{\text {bDepartment }}$ \\ of Ophthalmology, "Santo Spirito" Hospital, Pescara, Italy; 'Moorfields Eye Hospital, NHS \\ Foundation Trust, London, UK; ' Department of Ophthalmology, "Monfalcone-Gorizia \\ A.S.U.G.I.," Monfalcone, Italy; " Department of Ophthalmology, "De Gironcoli" Hospital, \\ Conegliano, Italy; fDepartment of Ophthalmology, "Villa Anna" Hospital, San Benedetto \\ del Tronto, Italy; " Department of Ophthalmology, "A. Murri" Hospital, Fermo, Italy; hPrivate \\ Practice "Dr. Latanza," Naples, Italy
}

\section{Keywords}

Choroidal neovascularization · Ocular tuberculosis · Choroidal granuloma

\begin{abstract}
An otherwise healthy 72-year-old Chinese patient diagnosed with exudative age-related macular degeneration and decreased vision in left eye was fully investigated. The retrospective analysis of past multimodal imaging revealed bilateral severe choroidal neovascularization and choroiditis associated with a positive tuberculin skin testing and interferon-gamma release assay (QuantiFERON-TB Gold - Cellestis ${ }^{\circledR}$, Chadstone, VIC, Australia) suggestive of latent ocular tuberculosis. The variable presentation and tests' results interpretation represent the greatest limitations in understanding and treating intraocular TB (IOTB). This may present without any other systemic symptoms, the intraocular tissues are of limited access to biopsies and other tests, including imaging and immunological tests, are of relative value. This case highlights how variable may be the presentation of IOTB, which can be easily misdiagnosed leading to a delayed treatment and worse prognosis.
\end{abstract}

\section{Karger ${ }^{\prime \prime}=$}




\section{Introduction}

Tuberculosis (TB) is one of the primary causes of morbidity and mortality worldwide. According to the WHO, about one-third of the world's population is infected by TB and $90 \%$ have latent TB [1]. More than 95\% of new infections occur in the developing countries especially in Africa and South Asia although an increasing number of TB infections have been reported also in developed countries due to multidrug-resistant TB, HIV, and global migration [2]. Intraocular TB (IOTB) is often not associated with clinical evidence of pulmonary TB, as up to $60 \%$ of extrapulmonary TB patients may not have respiratory system involvement. Moreover, IOTB may have a wide spectrum of presentations making diagnosis particularly difficult [3]. We report a case of IOTB misdiagnosed as age-related choroidal neovascularization $(\mathrm{CNV})$.

\section{Case Presentation}

A 72-year-old Chinese patient was referred to our clinic for a sudden visual loss in the left eye (OS). His history was significant for alcohol abuse and a diagnosis of bilateral exudative age-related macular degeneration made 18 months before and treated with 3 intravitreal injections of bevacizumab in both eyes (OU) at another hospital. However, he complained of progressive visual loss OU despite the therapy.

Our evaluation showed a best-corrected visual acuity of hand movement in the right eye (OD) and light perception OS. Anterior segment was significant for a white cataract OD. Posterior segment was unexplorable because of a white cataract OD and vitritis OS. Ocular B-mode ultrasonography revealed the presence of dense vitreous opacities associated with chorioretinal thickening OU (Fig. 1a, b).

A retrospective review of records acquired at the first presentation in another hospital was carried out. The first Spectral Domain-Optical Coeherence Tomography (SD-OCT) revealed active type I CNV OU with foveal and extra-foveal subretinal exudation and suspected bleeding associated to diffuse thickening of the choroid (Fig. 2a-d). Moreover, a round inferior-temporal lesion suggestive of choroidal granuloma (Fig. 2d) was observed OS. Fluorescein angiography (FA) and indocyanine green angiography (ICG) images of OD were of too low quality to be useful. FA in OS revealed a large area of hypofluorescence temporally to the macula consistent with retinal hemorrhage caused by active type I CNV (Fig. 2e, f). Inferiorly to this site, a hyper/hypofluorescent round lesion was also evident in the location of the suspected granuloma at ICG (Fig. 2g, h).
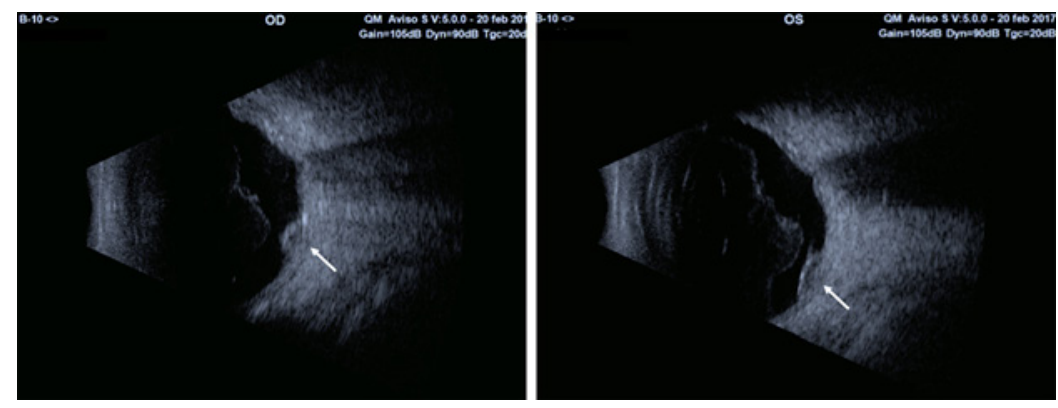

Fig. 1. B-mode ultrasonography (US). OD and OS B-mode US showing vitreous opacities and elevated chorioretinal lesions(white arrows). 


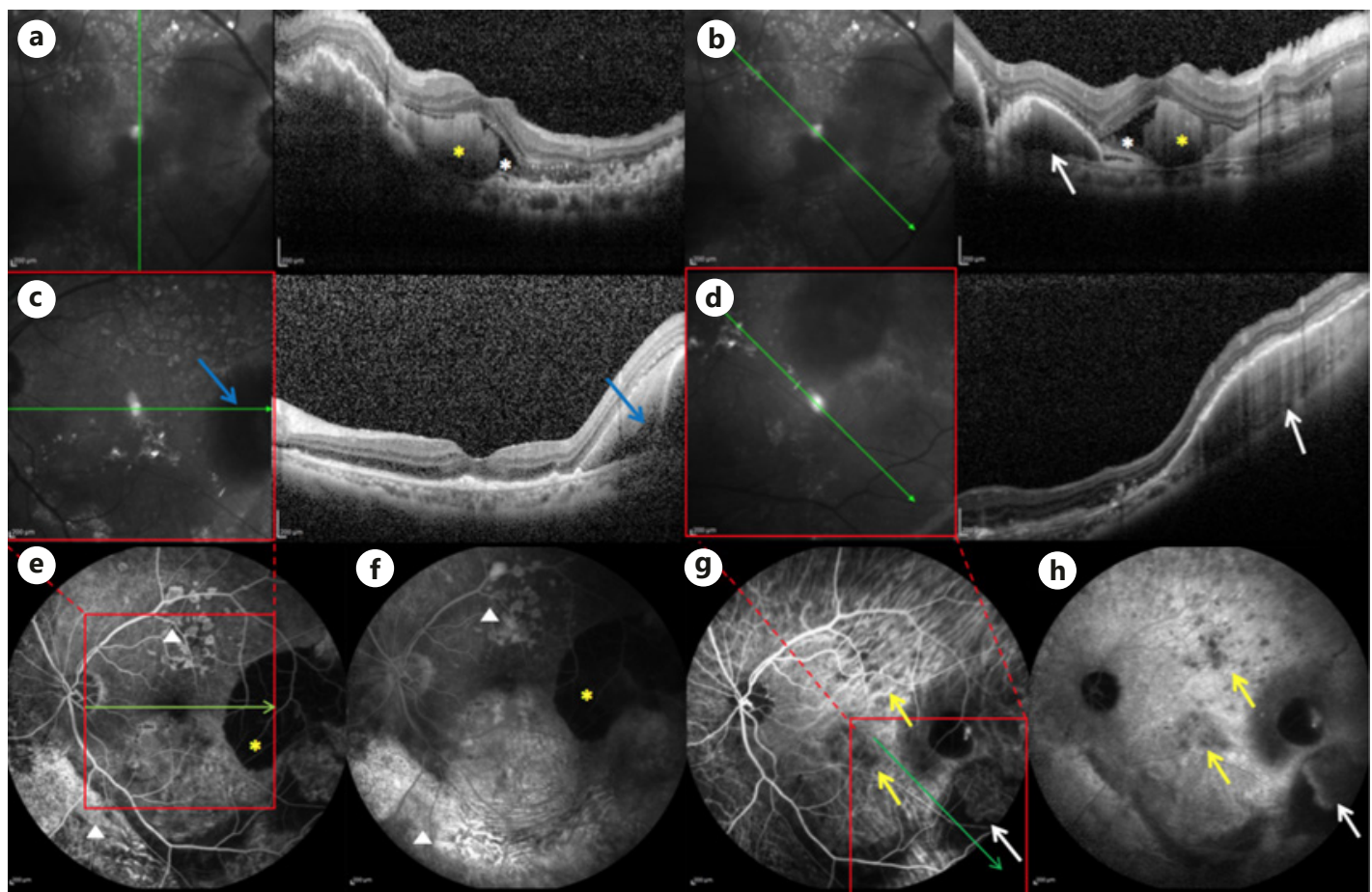

Fig. 2. Multimodal Imaging of the patient at the time of disease's onset (18 months before our observation). Infrared-SD-OCT shows lesions consistent with active CNV (white arrow), subretinal fluid (white asterisks), and suspected hemorrhage (yellow asterisks) in OD (a, b); infrared-SD-OCT OS shows perifoveal presumptive hemorrhagic detachment of pigmented epithelium (PED) (blue arrows) and a suspected granulomatous lesion inferiorly (white arrow) (c, d); red squares represent the magnified projection at infrared images of the correspondent area included by small red squares on FA (e) and ICG (g) images, while green arrow indicated the OCT scan direction; early and late phases of FA OS shows diffuse hypo/hyperfluorescent spots corresponding to atrophic changes of retinal pigmented epithelium (RPE) (white triangles) associated to perifoveal hypofluorescent lesion matching to the presumptive hemorrhagic PED (yellow asterisks) (e, f); early and late phases of ICG angiography OS discloses multiple hypocianescent dots (yellow arrows) suggestive of active choroiditis along with a granulomatous lesion appearing as round halfhypocianescent and halfhypercianescent lesion (white arrows) (g, h).

A full work-up to rule-out autoimmune diseases resulted negative as well as serology tests for HIV, syphilis and herpes virus infection. Tuberculin skin testing was markedly positive ( $>15 \mathrm{~mm}$ of induration) as well as interferon-gamma release assay (QuantiFERONTB Gold - Cellestis ${ }^{\circledR}$, Chadstone, VIC, Australia) while DNA analysis by PCR on aqueous humor was negative for M. tuberculosis. Pulmonary examination was unremarkable such as highresolution computed tomography (HRCT) of the chest. A diagnosis of possible bilateral IOTB [4] was established and antitubercular therapy (ATT) with isoniazid (250 mg/day), rifampin (450 mg/day), pyrazinamide (500 mg twice a day) and B6 vitamin was given in addition to oral prednisone $1 \mathrm{mg} / \mathrm{kg} /$ day started a week later and gradually tapered by the time. During following weeks, vitritis improved; 2 months later, pyrazinamide was suspended, cataract surgery was performed in OD and after 9 months of therapy, fundus examination disclosed an extensive residual subretinal fibrosis with no signs of active CNV (Fig. 3a, b) associated to a final VA of counting fingers OU. ATT (rifampin $450 \mathrm{mg} /$ day and isoniazid $250 \mathrm{mg} /$ day) associated with a maintenance dose of oral steroids $(12.5 \mathrm{mg} /$ day $)$ was continued up to 9 months of follow-up. 

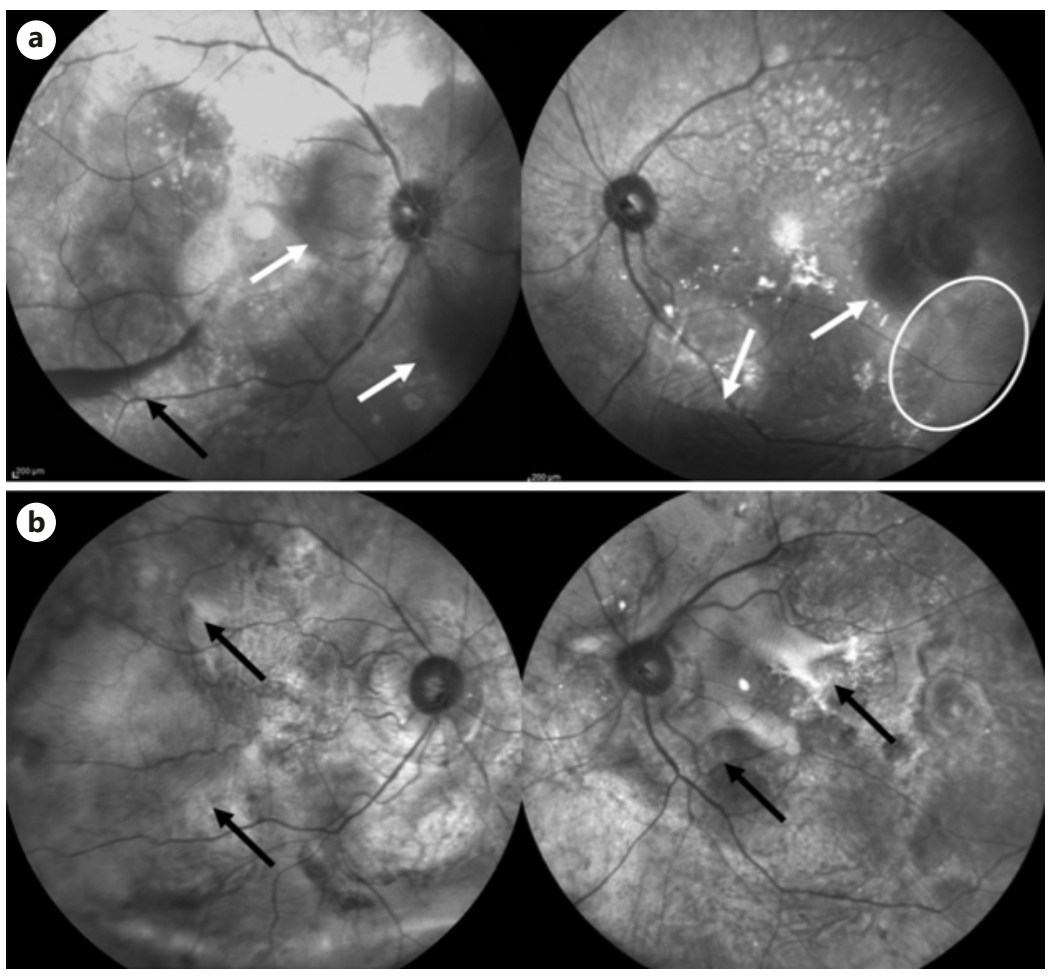

Fig. 3. Infrared imaging OU at baseline (a) and last follow-up (b). Infrared imaging OU at baseline shows extensive subretinal hemorrhages (white arrows), preretinal bleeding OD (black arrows) and suspected granulomatous lesion OS (white circle) (a); infrared imaging OU at last follow-up showing diffuse chorioretinal atrophy and residual fibrosis (black arrows) (b).

\section{Discussion}

IOTB may occur following primary infection, dissemination of systemic infection, reactivation of latent TB and immune-mediated disease. M. tuberculosis may cause infection in almost all ocular tissues, but posterior uveitis is the most common manifestation of the disease due to direct tissue infection or hypersensitivity response [3-7]. Although IOTBrelated uveitis presentation may be subtle, causing a delay in the diagnosis, the most common form is represented by disseminated choroiditis followed by focal choroiditis (unifocal choroidal lesion is more frequent than multiple lesions), while serpiginous-like choroiditis, neuroretinitis, vitritis, subretinal abscess, retinal vasculitis and optic neuritis are less common posterior segment manifestations [3-7]. IOTB-related choroiditis may present a multifocal pattern of variable lesions consisting in multiple foci of granuloma or a single elevated mass in the choroid often associated with overlying exudative retinal detachment [3-7]. Yu-Mei et al. [8] first described the association of choroidal TB with macular subretinal neovascularization in a middle-aged Chinese female with bilateral miliary choroidal TB. Since then, the association between CNV and ocular TB has been rarely reported by other authors $[9,10]$.

In our patient, $\mathrm{CNV}$ at the posterior pole and diffuse choroiditis $\mathrm{OU}$, with a suspected tuberculoma OS, represented the onset of disease. However, at first examination, CNV along with the patient's old age probably induced a diagnosis of exudative age-related macular degeneration, with subsequent decision of intravitreal anti-VEGF treatment in another hospital. The positivity of tuberculin skin and interferon-gamma release assay testing along with the slow and gradual response to therapy, supported the diagnosis of possible IOTB [4] 
in a patient migrated from an endemic area. Furthermore, the absence of pulmonary disease at HRCT of the chest did not rule out the possibility of IOTB in our case, considering that up to $60 \%$ of patients with extrapulmonary TB have neither clinical nor radiological evidence of pulmonary TB in cases of latent disease [3].

The diagnosis of IOTB is often difficult due to a wide spectrum of clinical presentations and the complexity of obtaining uveal biopsy for culture or histopathological examination to provide definitive proof of ocular infection with most reported cases remaining presumptive. One should keep in mind that tuberculous uveitis may occur in patients without signs or symptoms of systemic TB. Our case demonstrated that CNV may be a possible, albeit unusual, presentation of IOTB that should be fully investigated and ruled-out especially in patients from endemic areas. This unusual presentation should be remembered so as to allow a prompt diagnosis and an adequate therapy, which may be crucial for visual preservation.

\section{Statement of Ethics}

The patient involved in the present manuscript gave her written informed consent to publish the case (including publication of images).

\section{Conflict of Interest Statement}

The authors have no conflicts of interest to disclose.

\section{Funding Sources}

The present study was carried out with no fundings.

\section{Author Contributions}

E.I. as first and corresponding author made substantial contributions to the conception, the design of the work, the acquisition, analysis and interpretation of data, and the final revision of the paper; F.P., M.D., R.D., and A.P. made substantial contribution to the design and the final revision of the work; E.M., A.C., D.C., C.C., T.L., and A.Z. contributed by the acquisition, the analysis and the interpretation of the data, and the final revision of the paper. L.L. and C.P. made a significant contribution to the final revision of the paper.

\section{References}

1 Schlossberg D, Maher D, Raviglione MC. The global epidemic of tuberculosis: a World Health Organization perspective. In: Schlossberg D, editor. Tuberculosis and nontuberculous mycobacterial infections. 4th ed. Chapter 10. Philadelphia, PA: WB Saunders Company; 1999. p. 104-15.

2 WHO. Global tuberculosis control: key findings from the December 2009 WHO report. Wkly Epidemiol Rec. 2010;85(9):69-80.

3 Al-Shakarchi FI. Ocular tuberculosis: current perspectives. Clin Ophthalmol. 2015;9:2223-7.

4 Gupta A, Sharma A, Bansal R, Sharma K. Classification of intraocular tuberculosis. Ocul Immunol Inflamm. 2015 Feb;23(1):7-13.

5 Gupta V, Gupta A, Rao NA. Intraocular tuberculosis: an update. Surv Ophthalmol. 2007;52(6):561-87.

6 Gupta V, Shoughy SS, Mahajan S, Khairallah M, Rosenbaum JT, Curi A, et al. Clinics of ocular tuberculosis. Ocul Immunol Inflamm. 2015;23(1):14-24.

\section{Karger'}


7 Lee C, Agrawal R, Pavesio C. Ocular tuberculosis: a clinical conundrum. Ocul Immunol Inflamm. 2016;24(2): 237-42.

8 Yu-Mei C, Tse-Shiang Y, Shwn-Jiuan S, Jorn-Hon L. Macular subretinal neovascularization in choroidal tubercolosis. Ann Ophthalmol. 1989;21:225-9.

9 Bansal R, Bansal P, Gupta A, Gupta V, Dogra MR, Singh R, et al. Diagnostic challenges in inflammatory choroidal neovascular membranes. Ocul Immunol Inflamm. 2017;25(4):554-62.

10 Invernizzi A, Agarwal A, Di Nicola M, Franzetti F, Staurenghi G, Viola F. Choroidal neovascular membranes secondary to intraocular tuberculosis misdiagnosed as neovascular age-related macular degeneration. Eur J Ophthalmol. 2018;28(2):216-24. 